\title{
Eine systematische Untersuchung von der Wechselwirkung zwischen Wolframtrioxid Gassensoren und Luftfeuchtigkeit
}

\author{
Anna Staerz ${ }^{1}$, Mauro Epifani, Susanne Wicker ${ }^{1}$, Nicolae Barsan ${ }^{1}$ und Udo Weimar ${ }^{1}$ \\ ${ }^{1}$ Institut für Physikalische und Theoretische Chemie, Eberhard Karls Universität Tübingen/Deutschland \\ anna.staerz@ipc.uni-tuebingen.de \\ ${ }^{2}$ Consiglio Nazionale delle Ricerche-Istituto per la Microelecttronica e Microsistemi (CNR-Imm), \\ Lecce, Italy
}

\begin{abstract}
Zusammenfassung:
In früheren Untersuchungen hatte Wolframoxid $\left(\mathrm{WO}_{3}\right)$ sich als viel versprechend für die Atemgasanalyse erwiesen. Deshalb wurden in dieser Studie die Eigenschaften zweier $\mathrm{WO}_{3}$ Proben verglichen. Durch das Siebdruckverfahren wurden die $\mathrm{WO}_{3}$ Sensoren auf einem $\mathrm{Al}_{2} \mathrm{O}_{3}$-Substrat mit Platinelektrode gedruckt. Die Sensorsignale dieses Materials wurden gegenüber $\mathrm{CO}, \mathrm{NO}_{2}$, Toluol und Aceton für verschiedene Temperaturen zwischen $250{ }^{\circ} \mathrm{C}$ und $400{ }^{\circ} \mathrm{C}$ gemessen. Um den Einfluss von Luftfeuchtigkeit zu bestimmen, wurden die Messungen jeweils in trockener synthetischer Luft und in $30 \%$ und $80 \%$ relativer Feuchtigkeit $(\mathrm{RH})$ gemessen. Unsere Untersuchungen weisen darauf hin, dass insbesondere eines der beiden $\mathrm{WO}_{3}$ Materialien zur selektiven Detektion von Aceton in der Atemanalyse geeignet ist.
\end{abstract}

Schlagwörter: Gassensor, $\mathrm{WO}_{3}$, Atemgasanalyse, Acetone, und Widerstandsmessung

\section{Einleitung}

Bereits 1970 entdeckte Linus Pauling, dass der menschliche Atem mehr als 200 gasförmige Substanzen enthält [1]. Davon können viele als Biomarker dienen um Rückschlüsse auf den klinischen Zustand eines Patienten zu schließen. Atemgasanalyse könnte deshalb eine vielversprechende Methode sein Krankheiten zu diagnostizieren. Hierfür bieten sich anorganische Metalloxid-Halbleiter (MOX) Gassensoren an. Sie sind kompakt, günstig in der Herstellung und sensitiv [2]. Wolframtrioxid $\left(\mathrm{WO}_{3}\right)$ ist heute - neben $\mathrm{SnO}_{2}$ - eines der am wichtigsten Materialien in MOX Gassensoren [3]. Mehrere Publikationen haben hohe Sensorsignale von $\mathrm{WO}_{3}$ gegenüber Aceton beschrieben. Dies deutet auf eine vielversprechende Nutzung in der Atemgasanalyse hin. Eine Konzentrationsmessung von Aceton (1.76 - 3.73 ppm) im Atem [4] könnte eine Blutentnahme zur Blutzuckermessung vermeiden.

Obwohl ein $\mathrm{WO}_{3}$ Atemgasdetektorprototyp existiert [2], ist die Wechselwirkung zwischen Aceton und der $\mathrm{WO}_{3}$-Oberfläche unbekannt. Diese wurde hier mit DC-Widerstandsmessungen an verschiedenen $\mathrm{WO}_{3}$-Materialen untersucht. Die Luftfeuchtigkeit des menschlichen Atems ist gesättigt. Deshalb ist es wichtig die Wechselwirkung zwischen der
$W_{3}$-Oberfläche und der Luftfeuchtigkeit zu bestimmen. Alle Messungen wurden sowohl in trockener als auch in feuchter Luft durchgeführt. Bevor $\mathrm{WO}_{3}$ als Aceton-Sensor eingesetzt werden kann, muss die Wechselwirkung mit anderen relevanten Gasen untersucht werden. Beispielsweise enthält Atem eine erhöhte Konzentration von Toluol bei Lungenkrebs [5]. In dieser Studie wurde neben dem aromatischen Toluol, $\mathrm{NO}_{2}$ als ein Beispiel für ein oxidierendes Gas verwendet. $\mathrm{CO}$ wurde wegen seiner einfachen Molekülstruktur als ein Beispiel für ein reduzierendes Gas gemessen.

\section{Methoden und Materialien}

Sensorherstellung:

Das von Epifani et al. synthetisierte $\mathrm{WO}_{3}$ Pulver [6] wurde mit 1,2-Propandiol (Sigma Aldrich; 99,5+ \% A.C.S. Reagenz) zu einer Paste verarbeitet. Es wurde mittels Siebdruckverfahren auf ein $\mathrm{Al}_{2} \mathrm{O}_{3}$-Substrat mit einer Pt-Elektrode und ein Pt-Heizelement aufgetragen (Abb. 1). Die gassensitive $\mathrm{WO}_{3}$ Schicht wurde mehrere Stunden bei Raumtemperatur und über Nacht bei $70{ }^{\circ} \mathrm{C}$ getrocknet, dann in einem Rohrofen bei $400{ }^{\circ} \mathrm{C}$ für 10 Minuten, bei $500{ }^{\circ} \mathrm{C}$ für 10 Minuten und nochmals bei $400{ }^{\circ} \mathrm{C}$ für 10 Minuten kalziniert. 
Zum Vergleich wurde mit einem weiteren kommerziellen $\mathrm{WO}_{3}$-Pulver (Sigma-Aldrich > 100 nm Teilchengröße) ebenso verfahren.

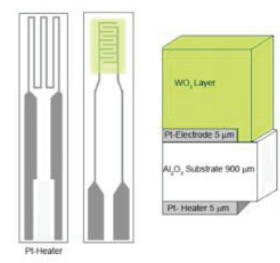

Abb. 1. Aufbau eines $\mathrm{WO}_{3}$-Sensors.

\section{Messungen}

Der Sensorwiderstand wurde mit einem Keithley 199 Elektrometer gemessen. Die verschiedenen Testgaskonzentrationen von $\mathrm{CO}, \mathrm{NO}_{2}$, Toluol und Aceton wurden mittels einer computergesteuerten Gasmischanlage mit Massenflussreglern bereitgestellt. Zweistündige Pulse von fünf verschiedenen Testgaskonzentrationen wurden gemessen. Zwischen den verschiedenen Testgasen wurde das System mit synthetischer Luft gespült. Das folgende Messprogramm wurde jeweils in trockener syn. Luft, $30 \% \mathrm{RH}$ und $80 \% \mathrm{RH}$ gemessen.

Tab. 1: Messprogramm für $D C$ -

Widerstandsmessungen in trockener syn. Luft.

\begin{tabular}{|l|l|l|l|}
\hline Zeit & Gas & Zeit & Gas \\
\hline$>5 h$ & syn. Luft & $>5 h$ & syn. Luft \\
\hline $2 h$ & $5 \mathrm{ppm} \mathrm{CO}$ & $2 h$ & $0,1 \mathrm{ppm}$ Toluol \\
\hline $2 h$ & $10 \mathrm{ppm} \mathrm{CO}$ & $2 h$ & $0,15 \mathrm{ppm}$ Toluol \\
\hline $2 h$ & $15 \mathrm{ppm} \mathrm{CO}$ & $2 h$ & $0,25 \mathrm{ppm}$ Toluol \\
\hline $2 h$ & $30 \mathrm{ppm} \mathrm{CO}$ & $2 h$ & $0,5 \mathrm{ppm}$ Toluol \\
\hline $2 h$ & $45 \mathrm{ppm} \mathrm{CO}$ & $2 h$ & $1,4 \mathrm{ppm}$ Toluol \\
\hline$>5 h$ & syn. Luft & $>5 h$ & syn. Luft \\
\hline $2 h$ & $0,75 \mathrm{ppm}$ Aceton & $2 h$ & $0,25 \mathrm{ppm} \mathrm{NO} 2$ \\
\hline $2 h$ & $1,5 \mathrm{ppm}$ Aceton & $2 h$ & $0,5 \mathrm{ppm} \mathrm{NO} 2$ \\
\hline $2 h$ & $2 \mathrm{ppm}$ Aceton & $2 h$ & $0,75 \mathrm{ppm} \mathrm{NO}_{2}$ \\
\hline $2 h$ & $2,5 \mathrm{ppm}$ Aceton & $2 h$ & $0,9 \mathrm{ppm} \mathrm{NO} \mathrm{NO}_{2}$ \\
\hline $2 h$ & $5 \mathrm{ppm}$ Aceton & $2 h$ & $2 \mathrm{ppm} \mathrm{\textrm {NO } _ { 2 }}$ \\
\hline
\end{tabular}

Diese Messungen wurden in $50^{\circ} \mathrm{C}$-Schritten in einem Temperaturbereich von $250-400^{\circ} \mathrm{C}$ durchgeführt. Es wurden je zwei $\mathrm{WO}_{3}$-Sensoren desselben Materials gleichzeitig vermessen. Das jeweilige Sensorsignal wurde mit der folgenden Formel für reduzierende Gase (inverses Verhältnis für oxidierende Gase) ermittelt.

$\mathrm{S}_{\text {ReduzierendesGas }}=\frac{\mathrm{R}_{\mathrm{O}}}{R_{\text {Reduzierendes Gas }}} \geq 1$

\section{Ergebnisse und Diskussion}

Die Messreihe bei $350{ }^{\circ} \mathrm{C}$ wird für die jeweiligen $\mathrm{WO}_{3}$ Proben gezeigt.

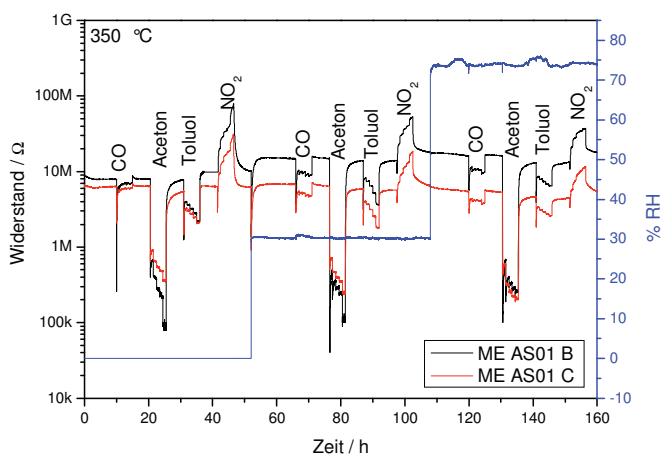

Abb. 2: $D C$-Widerstandsmessung bei $350^{\circ} \mathrm{C}$ von zwei Sensoren siebgedruckt mit WO3 Pulver von Epifani et al.

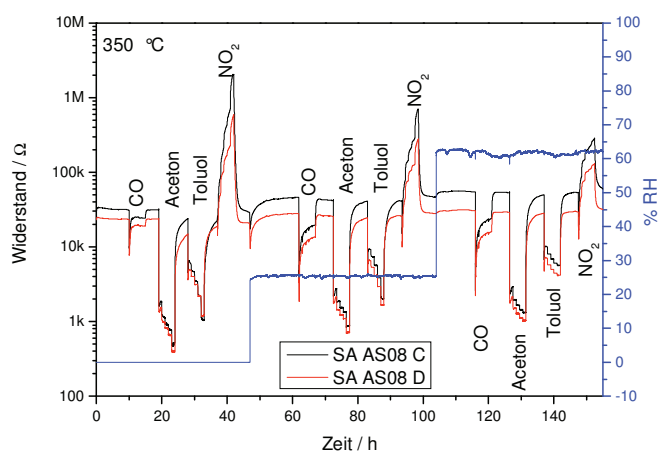

Abb. 3: DC-Widerstandsmessung bei $350{ }^{\circ} \mathrm{C}$ von zwei Sensoren siebgedruckt mit $\mathrm{WO}_{3}$ Pulver von Sigma Aldrich.

Wie die Messungen bei $350{ }^{\circ} \mathrm{C}$ beispielhaft zeigen, erhöht sich der Grundwiderstand, in beiden Fälle, mit steigender relativer Luftfeuchtigkeit. Dies steht im Gegensatz zu den Erwartungen, da Wasser in der Regel reduzierend wirkt [7]. Folglich liegt ein anderer Reaktionsweg zwischen der $\mathrm{WO}_{3}$-Oberfläche und Wasser vor, als normalerweise für ein n-typ Halbleiter angenommen wird. Dieser Trend ist bei den verschiedenen Temperaturen beobachtbar. Nicht nur die Grundwiderstände, sondern auch die Sensorsignale sind abhängig von der relativen Feuchtigkeit. Abb. 4 und 5 zeigen eine Übersicht über die Sensorsignale und dessen Abhängigkeit von der Feuchtigkeit. 


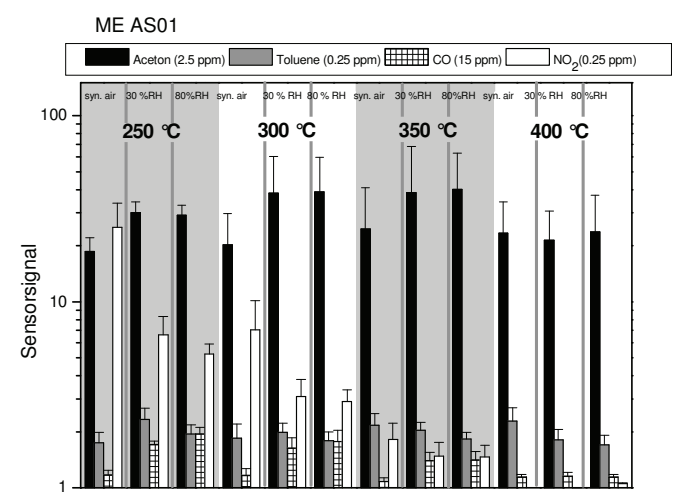

Abb. 4: Ein Übersicht die gemittelten Sensorsignale von zwei Sensoren siebgedruckt mit $\mathrm{WO}_{3}$ Pulver von Epifani et al.

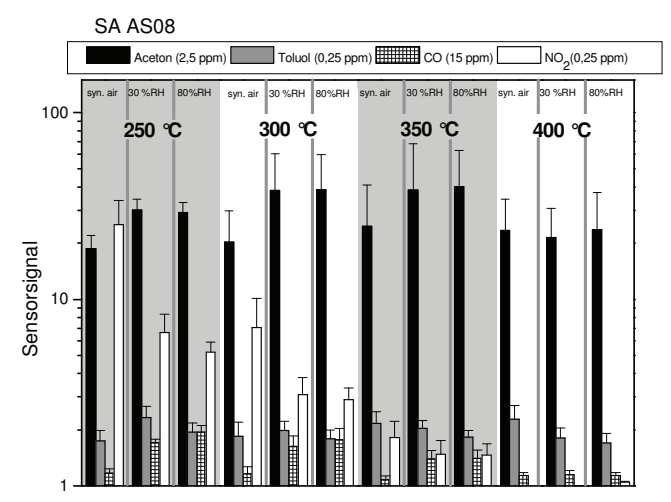

Abb. 5: Ein Übersicht die gemittelten Sensorsignale von zwei Sensoren siebgedruckt mit $W_{3}$ Pulver von Sigma-Aldrich.

Die Sensorsignale für $\mathrm{CO}$ sind in beiden Fälle niedrig, nehmen aber außer bei $400{ }^{\circ} \mathrm{C}$ mit steigender Feuchtigkeit zu. Ein gegenteiliges Verhalten ist für $\mathrm{SnO}_{2}$-Sensoren berichtet worden [7]. Diese Ergebnisse weisen darauf hin, dass Wasser nicht mit $\mathrm{CO}$ auf der $\mathrm{WO}_{3}$-Oberfläche konkurriert.

Die Sensorsignale für $\mathrm{NO}_{2}$ nehmen in beiden Fällen mit steigender Feuchtigkeit ab. Dies deutet drauf hin, dass Feuchtigkeit die Wechselwirkung zwischen $\mathrm{NO}_{2}$ und der Oberfläche hemmt. Bei der Probe von Epifani et al. sind die Signale für $\mathrm{NO}_{2}$ bei niedrigen Temperaturen hoch. Bei $350{ }^{\circ} \mathrm{C}$ sind die Sensorsignale für $\mathrm{NO}_{2}$ bei beiden Proben niedrig, während die Signale für Aceton hoch waren. Will man diese Sensoren selektiv zu Acetondetektion in menschlichen Atem verwenden, so scheint eine Messtemperatur von $350{ }^{\circ} \mathrm{C}$ gut geeignet zu sein.
Bemerkenswert ist außerdem, dass bei beiden Proben sind die absoluten Widerstandswerte für eine gegebene Acetonkonzentration in dem für die Atemanalyse relevanten Bereich (30 bis $80 \% \mathrm{RH}$ ) feuchtigkeitsunabhängig sind. Der Unterschied zwischen den Widerstandswerten liegt innerhalb der Fehlergrenze.

Tab. 2: Absolute Widerstandswerte für die Probe von Epifani et al.

\begin{tabular}{|l|l|l|}
\hline ME AS01 & $30 \% \mathrm{RH}$ & $80 \% \mathrm{RH}$ \\
\hline 0,75 & $605000 \pm 148492$ & $625000 \pm 63640$ \\
\hline 1,50 & $410000 \pm 70711$ & $355000 \pm 63640$ \\
\hline 2,00 & $340000 \pm 70711$ & $295000 \pm 49497$ \\
\hline 2,50 & $305000 \pm 77782$ & $255000 \pm 49497$ \\
\hline
\end{tabular}

Tab. 3: Absolute Widerstandswerte für die Probe von Sigma-Aldrich.

\begin{tabular}{|l|l|l|}
\hline SA AS08 & $30 \% \mathrm{RH}$ & $80 \% \mathrm{RH}$ \\
\hline 0,75 & $2440 \pm 438$ & $2560 \pm 537$ \\
\hline 1,50 & $1660 \pm 296$ & $1750 \pm 311$ \\
\hline 2,00 & $1400 \pm 226$ & $1455 \pm 261$ \\
\hline 2,50 & $1225 \pm 190$ & $1290 \pm 240$ \\
\hline
\end{tabular}

Aus diesem Grund könnten Acetonkonzentrationen direkt ohne Referenz gemessen werden. Die Probe von Epifani et al. scheint sich besser zum Detektieren von Aceton zu eignen, da bei $350{ }^{\circ} \mathrm{C}$ die Sensorsignale für Toluol niedriger als die der Sigma-Aldrich Probe sind.

Die Wechselwirkung zwischen der $\mathrm{WO}_{3}$-Oberfläche und weiteren Atemanalyse relevanten Gasen wird als Nächstes untersucht. Zudem wird die Wechselwirkung zwischen der Oberfläche und Feuchtigkeit mit diffusen Reflexions-Fouriertransformationsinfrarotspektroskopie (DRIFTS) Messungen untersucht. Um mögliche Mechanismen für die Wechselwirkung ableiten zu können.

\section{Literatur}

[1] L. Pauling , A. Robinson, R. Teranishi, P. Cary, Quantitative Analysis of Urine Vapor and Breath Gas by Gas-Liquid Partition Chromatography, Proceedings of the National Academy of Sciences of the United States of America, 10, 2374-2376 (1971); doi: 10.1073/pnas.68.10.2374

[2] M. Righettoni, A. Tricoli, S. Gass, A. Schmid, S. Pratsinis, A. Amann, Si: $\mathrm{WO}_{3}$ Sensors for Noninvasive Diabetes Diagnosis by Breath Analysis, Analytica Chimica Acta, 69-75 (2012); doi: 10.1016/j.aca.2012.06.002 
[3] M. Hübner, C. Simion, A. Haensch, N. Barsan,U. Weimar, CO Sensing Mechanism with $\mathrm{WO}_{3}$ Based Gas Sensors, Sensors and Actuators B,103-106, (2010); doi: 10.1016/j.snb.2010.09.040

[4] I. Lee, S. J. Choi, K. M. Park, S. S. Lee, S. Choi, I. D. Kim, C. O. Park, The Stability, Sensitivity and Response Transients of $\mathrm{ZnO}, \mathrm{SnO}_{2}$ and $\mathrm{WO}_{3}$ Sensors under Acetone, Toulene and $\mathrm{H}_{2} \mathrm{~S}$ Environments, Sensors and Actuators B, 300-307 (2014); doi: 10.1016/j.snb.2014.02.043

[5] K. I. Choi, S. Hwang, Z. Dai, Y. Kang, J. Lee, Supporting Information: Rh-Catalyzed $\mathrm{WO}_{3}$ with Anomalous Humidity Dependence of Gas Sensing Characteristics, Royal Society of Chemistry, 4, 53130-531369 (2014); doi: 10.1039/C4RA06654E

[6] M. Epifani, T. Andreau, J. Arbiol, R. Diaz, P. Siciliano, J. Morante, Chloro-Alkoxide Route to Transition Metal Oxides Synthesis of $\mathrm{WO}_{3}$ Thin Films and Powders from a Tungsten ChloroMethoxide,Chemistry of Materials, 14, 52155221 (2009); doi: 10.1016/j.aca.2012.06.002

[7] K. Grossmann, R. Pavelko, N. Barsan, U. Weimar, Interplay of $\mathrm{H}_{2}$, Water Vapor and Oxygenat the Surface of $\mathrm{SnO}_{2}$ Based Gas Sensors - An Operando Investigation Utilizing Deuterated Gases, Sensors and Actuators B: Chemical, 787-793 (2012); doi: 10.1016/j.snb.2012.03.075

[8] N. Barsan und U. Weimar, Conduction Model of Metal Oxide Gas Sensors, Journal of Electroceramics, 3, 143-147 (2001); doi: 10.1023/A:1014405811371 